\title{
Using a gene expression signature when controversy exists regarding the indication for adjuvant systemic treatment reduces the proportion of patients receiving adjuvant chemotherapy: a nationwide study
}

\author{
A. Kuijer, MD¹, A.C.M. van Bommel, MD², C.A. Drukker, MD, PhD³, M. van der Heiden-van der Loo, PhD, \\ C.H. Smorenburg, MD, PhD ${ }^{5}$, P.J. Westenend, MD, PhD ${ }^{6}$, S.C. Linn, MD ${ }^{5}$, E.J.Th. Rutgers, MD7, \\ S.G. Elias, MD, $\mathrm{PhD}^{8}$ and Th. van Dalen, $\mathrm{MD}, \mathrm{PhD}^{1}$
}

Purpose: The Dutch national guideline advises use of gene-expression signatures, such as the 70 -gene signature (70-GS), in case of ambivalence regarding the benefit of adjuvant chemotherapy (CT). In this nationwide study, the impact of 70-GS use on the administration of CT in early breast cancer patients with a dubious indication for $\mathrm{CT}$ is assessed.

Methods: Patients within a national guideline directed indication area for 70-GS use who were surgically treated between November 2011 and April 2013 were selected from the Netherlands Cancer Registry database. The effect of 70-GS use on the administration of CT was evaluated in guideline- and age-delineated subgroups addressing potential effect of bias by linear mixed-effect modeling and instrumental variable (IV) analyses.
Results: A total of 2,043 patients within the indicated area for 70-GS use were included, of whom 298 received a 70-GS. Without use of the $70-$ GS, $45 \%$ of patients received CT. The $70-$ GS use was associated with a $9.5 \%$ decrease in CT administration (95\% confidence interval (CI): -15.7 to $-3.3 \%)$ in linear mixed-effect model analyses and IV analyses showed similar results $(-9.9 \%$; $95 \% \mathrm{CI}:-19.3$ to -0.4$)$.

Conclusion: In patients in whom the Dutch national guidelines suggest the use of a gene-expression profile, 70-GS use is associated with a $10 \%$ decrease in the administration of adjuvant CT.

Genet Med advance online publication 19 November 2015

Key Words: adjuvant chemotherapy; breast cancer; gene-expression profiling; 70-gene signature; personalized medicine

\section{INTRODUCTION}

The mortality rates in breast cancer patients have decreased dramatically in the past two decades, due mainly to the administration and improvement of adjuvant systemic therapy. ${ }^{1,2}$ Clinical decision-making regarding administration of adjuvant systemic therapy in breast cancer patients is guided by conventional prognostic factors such as age, tumor size, tumor grade, status of axillary lymph nodes, and hormone receptor status. Clinicopathological risk indexes, such as Adjuvant!Online and the Nottingham Prognostic Index, use these factors to estimate the risk of recurrence and the benefit of adjuvant systemic treatment. ${ }^{3,4}$ Nevertheless, breast cancer tumors with comparable clinicopathological characteristics may have considerable different outcomes, reflecting the heterogeneity of the disease. ${ }^{5}$

Several gene expression profiles were developed to predict the risk of dissemination in breast cancer patients, and these gene expression profiles have drawn attention as an accurate alternative or adjunct for predicting outcome in individual breast cancer patients. The European Society of Medical Oncology suggests the use of gene expression profiles to gain additional prognostic and/or predictive information to complement pathology assessment, particularly in patients with estrogen receptor (ER)-positive (+) breast cancer. ${ }^{6}$ The current Dutch national (NABON) guideline for breast cancer (2012) recommends the use of a validated gene expression profile in patients with an invasive ductal carcinoma with ER-positive disease and a questionable indication for adjuvant chemotherapy (CT) based on conventional prognostic factors.

To date, research of gene expression profiles mainly focuses on the value of the test for the individual patient. The 70-gene signature (70-GS; MammaPrint) is the most commonly used gene expression profile in the Netherlands. ${ }^{8}$ The aim of the present study is to evaluate the impact of the 70-GS on the administration of adjuvant CT at a nationwide level in a subgroup of patients for whom the additional value of CT is debated and national guidelines suggest the use of a gene expression profile.

\footnotetext{
${ }^{1}$ Department of Surgery, Diakonessenhuis Utrecht, Utrecht, The Netherlands; ${ }^{2}$ Department of Surgery, Leiden University Medical Centre, Leiden, The Netherlands; ${ }^{3}$ Department of Surgery, Albert Schweitzer Hospital, Dordrecht, The Netherlands; ${ }^{4}$ Department of Research, Netherlands Comprehensive Cancer Organization (IKNL), Utrecht, The Netherlands; ${ }^{5}$ Department of Medical Oncology, Antoni van Leeuwenhoek Hospital-Netherlands Cancer Institute, Amsterdam, The Netherlands; ${ }^{6}$ Department of Pathology, Laboratory for Pathology Dordrecht, Dordrecht, The Netherlands; ${ }^{7}$ Department of Surgery, Antoni van Leeuwenhoek Hospital - Netherlands Cancer Institute, Amsterdam, The Netherlands; ${ }^{8}$ Department of Epidemiology, Julius Center for Health Sciences and Primary Care, University Medical Center Utrecht, Utrecht, The Netherlands. Correspondence: Thijs van Dalen (tvdalen@diakhuis.nl)
}

Submitted 20 April 2015; accepted 14 September 2015; advance online publication 19 November 2015. doi:10.1038/gim.2015.152 


\section{MATERIALS AND METHODS}

Data on patient, tumor, and treatment characteristics were obtained from the Netherlands Cancer Registry (NCR). The NCR is a nationwide database managed by the Netherlands Comprehensive Cancer Organization (IKNL) that prospectively registers clinicopathological and treatment characteristics of all cancer patients treated in the Netherlands since 1989. The NCR started registering the use of gene expression profiles in 2011. Between February 2007 and July 2011, the 70-GS became available in the Netherlands and was offered to patients enrolled in the Microarray in Node-Negative Disease May Avoid Chemotherapy (MINDACT) trial. ${ }^{9}$ In this trial patients were randomized between administration of adjuvant CT based on the gene signature or conventional prognostic clinicopathological factors.

Since accrual of the MINDACT trial ended, the 70-GS is increasingly used as an adjunct to conventional clinicopathological factors for clinical decision-making. It is the most widely used gene-expression profile in the Netherlands. Although OncotypeDX recently has become commercially available in the Netherlands, this gene-expression profile was not frequently used during the study period, and its use was therefore not taken into account.

\section{Study population}

Female patients with primary breast cancer who were older than 17 years of age, surgically treated between November 2011 and October 2013, and had no prior history of malignancy, neoadjuvant treatment, or distant metastasis upon diagnosis were identified in the NCR. According to the Dutch national guidelines, adjuvant $\mathrm{CT}$ should be administered to all lymph node-positive patients $(\geq \mathrm{N} 1 \mathrm{a})$ and to patients without lymph node involvement but with unfavorable clinicopathological tumor features (grade III tumors $>1 \mathrm{~cm}$, any tumor $>2 \mathrm{~cm}$, or HER2 + tumors) as well as to patients younger than 35 years of age. The current Dutch guideline advises against the administration of CT in patients who do not fulfill the aforementioned criteria, in patients more than 70 years of age, and in patients with grade I tumors smaller than $2 \mathrm{~cm}$.

According to the Dutch guidelines, "Validated gene expression profiles may be used in individual cases with a hormone receptor-sensitive invasive ductal carcinoma, if there is doubt about the indication for adjuvant chemotherapy on the basis of traditional prognostic factors." In line with this guideline we identified three groups of patients, all younger than age 70 years with ER-positive and HER2-negative invasive ductal carcinoma, where controversy exists regarding the administration of adjuvant CT: N0, grade I, $>2 \mathrm{~cm}$ (group A); N0, grade II, $>1 \mathrm{~cm}$ (group B); and N1mi, grade I/II (group C).

\section{Statistical analysis}

Frequencies of baseline characteristics were compared between patients who received a 70-GS versus patients who did not receive the test within the indicated area for 70-GS use, i.e., in whom controversy existed regarding the benefit of adjuvant
$\mathrm{CT}$, using a $\chi^{2}$ test for differences in categorical data. For normally distributed continuous variables (age and size), means were calculated and a $t$-test was performed. Concordance of the test result with the administration of CT in the overall study population and the aforementioned subgroups was assessed by dividing the sum of patients with a low-risk test result in whom adjuvant CT was omitted and patients with a high-risk test result who received adjuvant CT by all patients with a known test result.

For the whole group and the three categories of patients, the proportions receiving CT were assessed in relation to 70-GS use, and adherence to the low-risk or high-risk test result was evaluated. To provide the most valid results using observational data taking patient clustering in hospitals into account and to address potential "confounding by indication," linear mixedeffect regression models and instrumental variable (IV) analyses were used.

Linear mixed-effects regression analysis. The association between 70-GS use and the administration of adjuvant CT was assessed in a linear mixed model adjusting for possible observed confounders, taking into account patient clustering within hospitals. For this, we included both a random intercept per hospital (thus taking baseline differences in CT use among hospitals into account) and a random 70-GS slope per hospital (thus taking potential differences in the effect of the 70-GS on CT administration between hospitals into account). We adjusted for age and tumor size (continuously), and for grade, axillary status, and incidence year (categorically). Linearity of the relation between the continuous variables and CT use was inspected using a LOWESS smoother and was concluded to be linear for both age and size (the latter after truncation of $0.5 \%$ of the data above $5 \mathrm{~cm}$ ). Because the dependent variable (CT) was coded as 0 (no) and 1 (yes), the results of the linear mixed-effects analyses are on the risk difference scale, i.e., showing absolute differences in proportion of adjuvant CT use. The proportions of absolute differences were multiplied by 100 to present the results of these linear mixed-effects models as percentages in absolute risk differences. Besides investigating the association between $70-G S$ use on the administration of adjuvant $\mathrm{CT}$ in the overall study population, we assessed the effect of 70-GS within the aforementioned subgroups of patients $(\mathrm{A}, \mathrm{B}$, and $\mathrm{C})$ and age categories $(<50$ years, 50-59 years, and 60-69 years). To test for differences in the association between 70-GS and CT use between these subgroups, we calculated $P$ values for interaction by including interaction terms to the models.

IV analyses. In an attempt to optimally control confounding by indication and further assess the validity of the linear mixedeffect models, we performed IV analyses (two-stage least square (2SLS) using ordinary linear regression). Confounding by indication is a well-known phenomenon in studies with observational data, and it may be only partly resolved by multivariable regression analyses, such as mentioned above, 
because these methods cannot adjust for unmeasured confounders. An IV may serve as a substitute for randomization (pseudo-randomization) in non-randomized studies under the assumption that the IV (i) is strongly associated with the exposure (in our case 70-GS use), (ii) is unrelated to confounders, and (iii) has no direct association with outcome (the administration of adjuvant CT). ${ }^{10}$ We considered percentage of 70-GS use within the indicated area per hospital per year (IV 1), 70-GS use in the previous patient within the indicated area treated in the same hospital (IV 2), and a combination of both in the first stage of 2SLS analyses as IVs. All are measures for hospital 70GS preference. First, the association of these IVs with 70-GS use, the administration of adjuvant CT, or possible confounders was assessed by univariable logistic regression analysis. We considered using incidence year as an IV, however, incidence year was strongly related with CT use, also in patients treated in hospitals were the 70-GS was never used, and therefore failed the third assumption.

We present the results of the IV analyses with and without adjustment for the same potential confounders as in the aforementioned linear mixed-effects models (these were then included in both stages of the 2SLS approach). The results of the 2SLS analysis can be interpreted as the absolute change in percent CT use due to 70-GS use.

All $P$ values and 95\% confidence intervals (CIs) for the linear mixed-effects and the 2SLS analyses were based on 5,000-fold bootstrap resampling. A $P$ value $<0.05$ was considered statistically significant. All analyses were performed in R (version 3.1.3 for Mac OS) using the lme4 package (version 1.1-7) for the linear mixed-effects models.

\section{RESULTS}

Between November 2011 and October 2013, 2,043 women with primary breast cancer without prior malignancy or having received neoadjuvant treatment, within the indicated area for 70-GS use were surgically treated in the Netherlands. Of these patients, 298 (15\%) actually received 70-GS. Patients who received 70-GS were younger, had more limited axillary involvement, and suffered from smaller tumors compared with patients within the indicated area who did not receive 70-GS (Table 1). Furthermore, an increase in 70-GS use over time was observed.

\section{Adherence to test result}

The majority of patients were assigned to a low-risk test result (64\%), and high adherence rates to the 70-GS test result in the administration of adjuvant CT were observed in the overall study population $(86 \%)$. In the predefined guideline delineated subgroups $\mathrm{A}, \mathrm{B}$, and $\mathrm{C}$, the majority of the patients were assigned to the low-risk group by the $70-\mathrm{GS}(83,63$, and $63 \%$, respectively), and the administration of CT was in line with the 70 -GS result in 89,86 and $83 \%$ of the patients in subgroups A, B, and C, respectively (Table 2). The 70-GS did not affect the administration of adjuvant endocrine therapy within these subgroups.
Table 1 Patient and tumor characteristics according to 70 gene signature use in 2,043 patients within the indicated area for 70-GS use (all younger than 70 years of age with ER+/HER2-, invasive ductal carcinoma)

\begin{tabular}{|c|c|c|c|}
\hline & $\begin{array}{c}\text { 70-GS used, } \\
n=298, n(\%)\end{array}$ & $\begin{array}{l}\text { 70-GS not used, } \\
n=1,745, n(\%)\end{array}$ & $P$ value \\
\hline \multicolumn{4}{|c|}{ Patient characteristics } \\
\hline $\begin{array}{l}\text { Age at diagnosis in } \\
\text { years, mean }(\mathrm{SD})^{\mathrm{a}}\end{array}$ & $55.6(8.3)$ & $56.2(8.8)$ & 0.24 \\
\hline$<50$ & $78(26)$ & $415(24)$ & \\
\hline $50-69$ & $220(74)$ & $1,330(76)$ & 0.41 \\
\hline \multicolumn{4}{|l|}{ Incidence year } \\
\hline 2011 & $18(6)$ & $227(13)$ & \\
\hline 2012 & $189(63)$ & $1,252(72)$ & \\
\hline 2013 & $91(31)$ & $266(15)$ & $<0.001$ \\
\hline \multicolumn{4}{|l|}{ Tumor characteristics } \\
\hline \multicolumn{4}{|c|}{ Pathological axillary status (pN) } \\
\hline pNO (i-/i+) & $238(80)$ & $1,346(77)$ & \\
\hline pN1mi & $60(20)$ & $399(23)$ & 0.33 \\
\hline \multicolumn{4}{|c|}{ Pathological tumor size $(\mathrm{cm})$} \\
\hline Mean $(S D)^{a}$ & $1.7(0.6)$ & $1.8(0.8)$ & 0.01 \\
\hline$\leq 2$ & $230(77)$ & $1214(70)$ & \\
\hline$>2$ & $68(23)$ & $531(30)$ & $<0.01$ \\
\hline \multicolumn{4}{|l|}{ Invasive tumor grade } \\
\hline Grade I & $41(14)$ & $253(14)$ & \\
\hline Grade II & $257(86)$ & $1,492(86 \%)$ & 0.80 \\
\hline
\end{tabular}

ER, estrogen receptor; pNO (i-li+), no axillary lymph node involvement or isolated tumor cells; pN1 mi, micro-metastases.

a-test; other data represent $\chi^{2}$ test values.

\section{Linear mixed-effects regression analysis}

In the group of patients where the 70-GS was considered indicated according to the national guideline $(n=2,043)$, use of the $70-\mathrm{GS}$ was associated with a $9.5 \%$ (95\% CI: -15.7 to -3.3 ; $P=0.003)$ absolute reduction in the administration of CT after adjustment for measured confounders in linear mixedeffect model analyses (Table 3 ). A younger age, intermediate invasive tumor grade, and micro metastatic involvement of the axillary lymph nodes showed a strong positive association with the administration of adjuvant CT (Supplementary Table S1 online).

Subgroup analyses revealed a significant reduction in the administration of adjuvant CT after 70-GS use in subgroups A and C (-24.0\%; 95\% CI: -46.5 to $-1.6 ; P=0.035$ and $-16.0 \%$; $95 \%$ CI: -28.0 to $-4.0 ; P=0.009$, respectively) but no significant reduction in subgroup B (-5.8\%; 95\% CI: -13.0 to 1.3 ; $P=0.11)$. Although the association between $70-$ GS use and the administration of adjuvant $\mathrm{CT}$ is lower in subgroup $\mathrm{B}$ than in subgroup $\mathrm{A}$, this difference in association was not significant ( $P$ for interaction $70-G S^{\star}$ group B: 0.122; Figure 1).

The strongest relation between 70 -GS use and the administration of adjuvant CT was seen in younger patients. In patients younger than 50 years of age, a $26.9 \%$ reduction in the administration of CT was observed in patients who received $70-\mathrm{GS}$ (95\% CI: -38.3 to $-15.6 ; P<0.001$ ). 
In patients between 50 and 59 years of age, 70-GS use led to a $9.2 \%$ reduction in the administration of adjuvant CT; however, this was not significant $(95 \% \mathrm{CI}:-18.7$ to $0.3 ; P=$ 0.058; Table 3 ). In the older age category a reverse relation is observed: $70-\mathrm{GS}$ use resulted in a $3.5 \%$ (95\% CI: -5.9 to

Table 2 Administration of adjuvant CT and ET in patients within the indicated area for 70-GS use who received the 70-GS, i.e., in whom controversy exists regarding the administration of adjuvant CT based on conventional prognostic factors

\begin{tabular}{llccc}
\hline & & N & CT (\%) & ET (\%) \\
\hline All patients & 70-gene signature & 298 & 34 & 90 \\
(Groups A, B, and C & Low risk & 169 & 10 & 88 \\
combined) & High risk & 95 & 80 & 91 \\
& Risk unknown & 34 & 24 & 97 \\
& Adherence to test result & $86 \%$ & - & - \\
\hline Group A & 70-gene signature & 19 & 21 & 100 \\
(N0, grade I, > 2cm) & Low risk & 15 & 7 & 100 \\
& High risk & 3 & 67 & 100 \\
& Risk unknown & 1 & 100 & 100 \\
& Adherence to test result & $89 \%$ & - & - \\
\hline Group B & 70-gene signature & 220 & 34 & 89 \\
(N0, grade II, >1 cm) & Low risk & 120 & 8 & 88 \\
& High risk & 72 & 76 & 88 \\
& Risk unknown & 28 & 36 & 96 \\
& Adherence to test result & $86 \%$ & - & - \\
& 70-gene signature & 59 & 39 & 88 \\
Group C & Low risk & 34 & 18 & 82 \\
(pN1 mi, grade l/II) & High risk & 20 & 85 & 100 \\
& Risk unknown & 5 & 0 & 80 \\
& Adherence to test result & $83 \%$ & & \\
\hline
\end{tabular}

CT, chemotherapy; ET, adjuvant endocrine therapy; GS, gene signature. Adherence to risk profile $=$ proportion of patients with a low-risk test result and omission of CT or a high-risk test result and administration of CT of all patients with known 70-GS test results.
12.9; $P=0.48)$ increase in the administration of adjuvant CT ( $P$ for interaction $70-G S^{\star} 60-69$ year of age: $<0.001$; see Supplementary Figure S1 online).

\section{IV analysis}

To assess the validity of the two IVs as an instrument in this study, we confirmed that both percentage of 70-GS use within the indicated area per hospital per year (IV1) and use of the 70-GS in the previous patient within the indicated area (IV2) had a strong positive association with 70-GS use. The 70-GS was

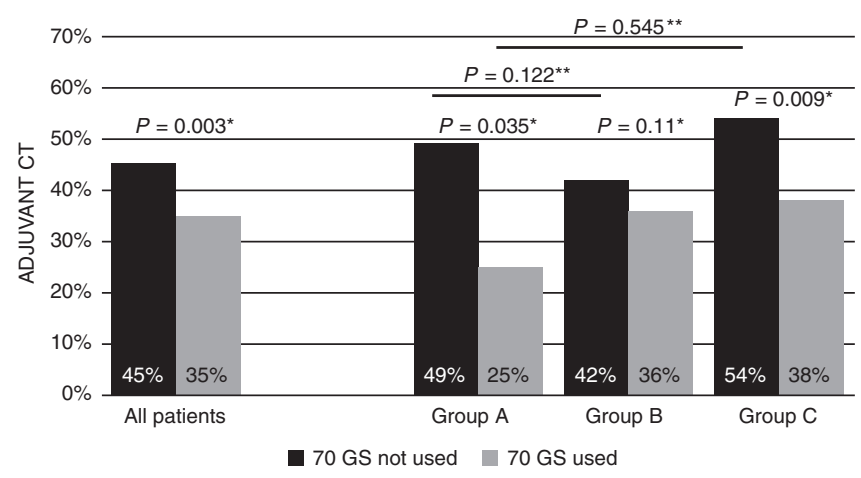

Figure 1 Absolute risk of receiving adjuvant CT according to the (fully adjusted) linear mixed model in guideline-delineated subgroups of patients within the indicated area for 70-GS use. All patients younger than 70 years of age with ER+/HER2- invasive ductal carcinoma (subgroup A: NO, BRI, >2 cm; subgroup B: NO, BRII, >1 cm; subgroup C: N1mi, BRI/II). $\mathrm{CT}$, chemotherapy; 70-GS, 70-gene signature. ${ }^{*}$ Calculated using the fully adjusted linear mixed model; age, tumor size, invasive tumor grade, axillary involvement, and incidence year were included as covariables in the model. Projected values are the percent chemotherapy use for those without and with the 70-GS as derived from the linear mixed models' regression equations filled in at the mean of all other covariables for the entire (sub-)group (i.e., the projected difference between 70-GS use and non-use is adjusted for confounding). ${ }^{* * P} P$ values for interaction between $70-$ GS use and the subgroups.

Table 3 The effect of 70-GS use on the administration of adjuvant CT as observed with linear mixed-effect modeling, in the overall study population $(n=2,043)$, guideline- and age-delineated subgroups of patients within the indicated area for 70-GS use, with and without adjustment for potential confounders

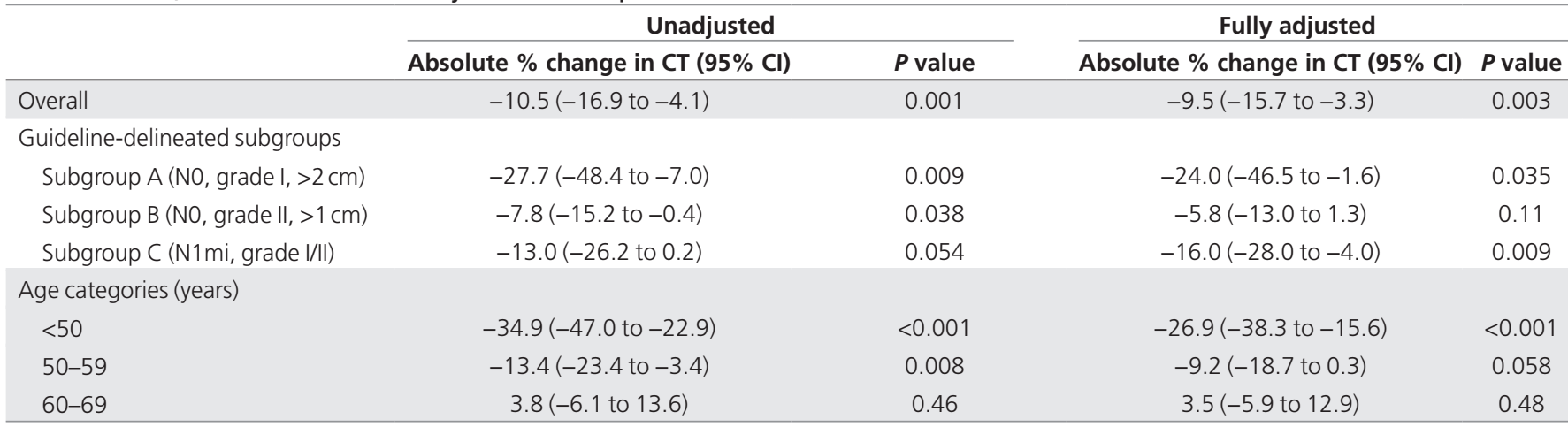

$P$ values for interaction between 70-GS use and subgroups: subgroup 70-GS*group B: $P=0.122 ; 70-G S *$ group $C: P=0.545$. $P$ values for interaction between 70-GS use and age categories; 70-GS*50-69 years of age: $P=0.017 ; 70-G S * 60-69$ years of age: $P<0.001$. Age (per year), incidence year (2011, 2012, or 2013), size (per mm), axillary involvement (NOi-/i+ vs. N1 mi), and invasive tumor grade (grade I vs. II) were included in the fully adjusted model as covariables.

$\mathrm{Cl}$, confidence interval. 
Table 4 The effect of 70-GS use on the administration of adjuvant CT in 2,043 patients within the indicated area for 70GS use as derived from different instrumental variable (IV) analyses (two-stage least-square regression analysis)

\begin{tabular}{|c|c|c|c|c|}
\hline & \multicolumn{2}{|l|}{ Unadjusted } & \multicolumn{2}{|l|}{ Fully adjusted } \\
\hline & Absolute $\%$ change in $\mathrm{CT}(95 \% \mathrm{Cl})$ & $P$ value & Absolute \% change in CT $(95 \% \mathrm{Cl})$ & $P$ value \\
\hline IV 1 & $-44.1(-75.0$ to -13.2$)$ & 0.005 & $-27.6(-54.5$ to -0.6$)$ & 0.045 \\
\hline IV 2 & $-20.6(-31.2$ to -10.0$)$ & $<0.001$ & $-10.2(-19.6$ to -0.8$)$ & 0.033 \\
\hline
\end{tabular}

70-GS use in the previous patient within the indicated area (IV 1), percentage of 70-GS use within the indicated area per hospital per year (IV 2), and a combination of both were used as IVs. Age (per year), incidence year (2011, 2012, or 2013), size (per mm), axillary involvement (NOi-/i+ vs. N1 mi), and invasive tumor grade (grade I vs. II) were included in the fully adjusted model as covariables.

$\mathrm{Cl}$, confidence interval; $\mathrm{CT}$, chemotherapy; 70-GS, 70-gene signature.

used in $5.6 \%$ of the patients within the indicated area in hospitals that less frequently used $70-$ GS $(0-25 \% 70-$ GS use within the indicated area per year), which was $47.9 \%$ for patients treated in hospitals who used $70-\mathrm{GS}$ in $>25 \%$ of the patients within the indicated area (odds ratio: 14). If the 70-GS was used in the previous patient within the indicated area, then $33.6 \%$ of the subsequently treated patients also received a 70-GS compared with $12.2 \%$ if the prior patient did not receive a 70-GS (odds ratio: 18). Combining IV1 and IV2 in the first stage of the 2SLS regression method showed the strongest association with 70-GS use (5.2 vs. $45.3 \%$; odds ratio: 22 ; comparing the best scoring $25 \%$ of patients for this IV vs. the rest). Both IV1 and IV2 were generally less associated with potential confounders (age at diagnosis and tumor characteristics) than actual 70-GS use. However, incidence year was not equally distributed between levels of the three IVs (Supplementary Table S2 online).

The results of the IV analyses are shown in Table 4, with and without adjustment for potential confounders. The analyses of both IV1 and IV2 revealed a significant reduction in the administration of adjuvant $\mathrm{CT}$ in patients who received a 70-GS (-27.6\%; 95\% CI: -54.5 to $-0.6 \%$; $P=0.045$ and $-10.2 \%$; $95 \%$ CI: -19.6 to $-0.8 ; P=0.033$, for IV 1 and 2 , respectively). The results of the IV analysis with the combined IVs were similar to the results of the adjusted linear mixed-effects regression model $(-9.9 \%$; $95 \%$ CI: -19.3 to $-0.4 ; P=0.040)$.

\section{DISCUSSION}

In this nationwide study, patients in whom the 70-GS was used received $10 \%$ less adjuvant CT compared with patients who did not receive the 70-GS in a cohort of Dutch breast cancer patients for whom controversy exists regarding the benefit of CT based on clinicopathological characteristics. Furthermore, in this selection of patients, compliance with the test result was high.

We observed a significant and clinically relevant decrease in the administration of adjuvant CT after use of the 70-GS in patients with an uncertain indication for adjuvant CT based on conventional prognostic factors after correction of all measured confounders. Currently, there is only limited evidence concerning the impact of the 70-GS on the administration of CT. Various studies have reported the impact of gene expression profiles within cohorts of patients who received the test. ${ }^{11,12}$ A theoretical change in adjuvant systemic treatment decisions was reported in $\sim 30 \%$ of patients after use of the $70-\mathrm{GS}$, resulting in a more tailored adjuvant systemic treatment plan for the individual patient. ${ }^{13}$ Current evidence is based on a few impact studies with relatively small numbers of patients, predominantly describing a hypothesized decrease in the administration of adjuvant CT after 70-GS use in early breast cancer patients. ${ }^{12,14}$ Similar results were reported regarding the impact of the 21-gene Recurrence Score (OncotypeDx) on the administration of adjuvant CT. ${ }^{15-17}$ In addition, high compliance rates with the test results in patients with early-stage ER+, node-negative breast cancer have been reported. ${ }^{18}$ Yet, to our knowledge, there are no nationwide studies assessing the impact on the administration of adjuvant CT for either OncotypeDx or the 70-GS.

Recently, Cusumano et al. conducted a European interinstitutional impact study of the 70-GS $(n=194)$. Cases were presented to clinician panels in four different countries asking for an adjuvant treatment advice, first without and then with knowledge of the 70-gene test result. In the subset of ER-positive and HER2Neu-negative patients $(n=100)$, a $21 \%$ (73 vs. $52 \%$ ) expected absolute decrease in the administration of adjuvant CT was reported when the 70-GS result was taken into account by a Dutch multidisciplinary team. ${ }^{19}$ Our results are in line with this report, although we saw a smaller decrease in the administration of adjuvant CT when the 70-GS was used. This difference may be explained by differences in the design of the present study in which patients were categorized by a combination of hormone status, HER2-status, tumor size, differentiation grade, and lymph node involvement. In addition, the smaller proportion of patients receiving adjuvant $\mathrm{CT}$ in the control group in our study (44 vs. $73 \%$ ) illustrates the different national attitudes toward the administration of adjuvant CT in the absence of a gene signature test. In fact, the relative reduction of administered adjuvant CT was comparable between our population-based study and Cusumano's questionnaire study.

Approximately $45 \%$ of patients in whom controversy exists regarding the administration of adjuvant CT received adjuvant CT without use of the 70-GS. This reflects the current controversy regarding the administration of adjuvant CT in these patients. High compliance rates with the 70-GS test result were seen demonstrating the propensity to adhere to a reproducible advice in this group of patients. The 70-GS assigned the majority of these patients to the low risk category ( $83 \%$ in group A, $63 \%$ in group B, and $63 \%$ in group C). In particular, in group 
$\mathrm{B}$, consisting of patients with grade II breast cancer, the proportion of low-risk test results was higher than reported in previous studies assigning $\sim 50 \%$ to the high risk category and $50 \%$ to the low risk category. ${ }^{20}$ Especially in subgroup A (N0, BRI, $>2 \mathrm{~cm}$ ) and subgroup C (N1mi, BRI/II), 70-GS use was associated with less administered CT, whereas in subgroup B (N0, BRII, >1 cm) only a non-significant trend was observed. Nevertheless, the $P$ values for interaction between the subgroups indicate that the effect of 70-GS use on the administration of CT does not significantly differ between the various subgroups. Presumably, also considering the prevalence of genomic high-risk patients, the current sample size of subgroup B is too small to reach significance.

This is the first nationwide study to report the independent association between the use of the 70-GS and the administration of adjuvant CT in patients for whom controversy exists according to the national guidelines, i.e., patients with ER-positive, HER2-negative invasive ductal tumors of low or intermediate malignancy grade in the absence of overt lymph node metastases. This was supported by linear mixed-effect modeling, which has as its main advantage that no independence is assumed among observations, allowing correlated observations within a unit or cluster. Because hospital preference or even clinician preference plays an important role in 70-GS use and the administration of adjuvant CT, patient clustering might have influenced the results. Therefore, this statistical approach is ideally suited for assessment of the association between 70-GS use and the administration of adjuvant $\mathrm{CT}$ and makes the observed association more valid. In this study, other factors also influenced the decision to administer CT in the subset of patients where CT was considered controversial. In particular, the effect of age was remarkable. The high proportion of young women who received adjuvant CT in the controversy group reflects an understandably more aggressive attitude in younger women. Nonetheless, although the overwhelming majority of younger women received adjuvant CT when 70-GS was not used (83\%; Supplementary Figure S2 online), 53\% of these young women received adjuvant CT when 70-GS was used, resulting in the largest relative and absolute reduction of administered CT. By contrast, in the older age category (60-69 years of age), 70-GS use was associated with an increased administration of adjuvant CT (Supplementary Figure S2). Although this effect in the older age category is not statistically significant in the linear mixed-effect analyses, the $P$ value for interaction $(P<0.001)$ implies that the effect of 70-GS use on the administration of CT in older patients is different from those in patients younger than 50 years of age. These findings might indicate that the physician's intention for 70-GS use is associated with age, but the retrospective observational design of the current study precludes detailed analyses of these findings.

Confounding by indication is a well-known phenomenon in studies using observational data. In an attempt to control this form of bias, we performed additional IV analyses. ${ }^{10}$ An IV is an external factor that influences outcome (in our case the administration of CT) exclusively through its effect on exposure (70-GS use), unrelated to potential confounders. We decided to use facility-prescribing patterns as IVs, an approach adopted previously by others. ${ }^{20}$ In our case, a high-quality IV should be strongly related to 70-GS use, which was the case when combining both IVs. Furthermore, a robust IV should not be associated with other confounders of the administration of CT. Because confounders can be measured or unmeasured, it is impossible to assess the direct relation between an IV and all possible confounders. Therefore, most authors using IVs report on the relation between IVs and measured confounders (for example, patient characteristics) under the assumption that if the IV is not related to a measured confounder, it likely is also not related to unmeasured confounders. ${ }^{21}$ In our case, all IVs (IV1: 70-GS use in the previous patient within the indicated area; IV2: proportion of 70-GS use per hospital within the indicated area) and a combination of both resulted in a better balance for measured confounders compared with actual 70-GS use (Supplementary Table S2 online), but a disbalance remained for the variable incidence year. Therefore, we also adjusted the IV effects for the measured potential confounders, and the combined IV analysis resulted in a strikingly similar absolute risk reduction for the administration of CT after 70-GS as observed in the standard linear mixed-effect regression analyses.

The prognostic value of the 70-GS has been validated in multiple retrospective and one prospective patient series. ${ }^{8,13,22-25}$ Evidence supporting the predictive value of the 70-GS on CT benefit is limited; therefore, its clinical utility mainly lies in its prognostic capacity. ${ }^{26-28}$ Currently, the MINDACT trial is being conducted and the first results are expected by the end of this calendar year, which will further assess the predictive capacity of the 70-GS in a randomized prospective setting. ${ }^{9}$ In the present study, i.e., in patients who were treated within the indicated area for gene expression profiles characterized as having a fairly good prognosis, it was the prognostic value of the 70-GS that was used to discern a group of patients with such good outcome that no substantial benefit was to be expected of adjuvant CT. The observed effect in the present study is not attributable to a hitherto unproven predictive value of the 70-GS.

In conclusion, in Dutch early-stage breast cancer patients with a dubious indication for adjuvant CT based on clinicopathological factors, $70-$ GS use is associated with a $10 \%$ decrease in the administration of adjuvant CT.

\section{SUPPLEMENTARY MATERIAL}

Supplementary material is linked to the online version of the paper at http://www.nature.com/gim

\section{ACKNOWLEDGMENT}

This work was supported by the Dutch Cancer Society (KWF) grant number: DU 2014-7131.

\section{DISCLOSURE}

The authors declare no conflict of interest. 


\section{REFERENCES}

1. Early Breast Cancer Trialists' Collaborative Group (EBCTCG). Systemic treatment of early breast cancer by hormonal, cytotoxic, or immune therapy: 133 randomized trials involving 31000 recurrences and 24000 deaths among 75 000 women. Lancet 1992;339:71-85.

2. Early Breast Cancer Trialists' Collaborative Group (EBCTCG). Effects of chemotherapy and hormonal therapy for early breast cancer recurrence and 15-year survival overview of the randomized trials. Lancet 2005;365:1687-717.

3. Ravdin PM, Siminoff LA, Davis GJ, et al. Computer program to assist in making decisions about adjuvant therapy for women with early breast cancer. J Clin Oncol 2001;19:980-991.

4. Galea MH, Blamey RW, Elston CE, Ellis IO. The Nottingham Prognostic Index in primary breast cancer. Breast Cancer Res Treat 1992;22:207-219.

5. Mook S, Van't Veer LJ, Rutgers EJ, Piccart-Gebhart MJ, Cardoso F. Individualization of therapy using Mammaprint: from development to the MINDACT trial. Cancer Genomics Proteomics 2007;4:147-155.

6. Senkus E, Kyriakides S, Penault-Llorca F, et al. Primary breast cancer: ESMO Clinical Practice Guidelines for diagnosis, treatment and follow-up. Ann Oncol 2013;24(suppl 6):7-23.

7. Kwaliteitsinstituut voor de gezondheidszorg CBO VvIK. Risicoprofilering. Richtlijn mammacarcinoom. 2013;81-83.

8. van de Vijver MJ, He YD, van't Veer LJ, et al. A gene-expression signature as a predictor of survival in breast cancer. N Eng J Med 2002;347:1999-2009.

9. Rutgers E, Piccart-Gebhart MJ, Bogaerts J, et al. The EORTC 10041/BIG 03-04 MINDACT trial is feasible: results of the pilot phase. Eur J Cancer 2011;47:27422749.

10. Greenland S. An introduction to instrumental variables for epidemiologists. Int J Epidemiol 2000;29:722-729.

11. Torrisi R, Garcia-Etienne CA, Losurdo A, et al. Potential impact of the 70-gene signature in the choice of adjuvant systemic treatment for ER positive, HER2 negative tumors: a single institution experience. Breast 2013;22:419-424.

12. Exner R, Bago-Horvath Z, Bartsch R, et al. The multigene signature MammaPrint impacts on multidisciplinary team decisions in ER+, HER2- early breast cancer. $\mathrm{Br}$ J Cancer 2014;111:837-842.

13. Drukker CA, Bueno-de-Mesquita JM, Retèl VP, et al. A prospective evaluation of a breast cancer prognosis signature in the observational RASTER study. Int J Cancer 2013;133:929-936.

14. Drukker CA, van den Hout HC, Sonke GS, et al. Risk estimations and treatment decisions in early stage breast cancer: agreement among oncologists and the impact of the 70-gene signature. Eur J Cancer 2014;50:1045-1054.

15. Albanell J, González A, Ruiz-Borrego M, et al. Prospective transGEICAM study of the impact of the 21-gene Recurrence Score assay and traditional clinicopathological factors on adjuvant clinical decision making in women with estrogen receptor-positive (ER+) node-negative breast cancer. Ann Oncol 2012;23:625-631.

16. Ademuyiwa FO, Miller A, O'Connor T, et al. The effects of oncotype DX recurrence scores on chemotherapy utilization in a multi-institutional breast cancer cohort. Breast Cancer Res Treat 2011;126:797-802.

17. Partin JF, Mamounas EP. Impact of the 21-gene recurrence score assay compared with standard clinicopathologic guidelines in adjuvant therapy selection for node-negative, estrogen receptor-positive breast cancer. Ann Surg Oncol 2011;18:3399-3406.

18. McVeigh TP, Hughes LM, Miller N, et al. The impact of Oncotype DX testing on breast cancer management and chemotherapy prescribing patterns in a tertiary referral centre. Eur J Cancer 2014;50:2763-2770.

19. Cusumano PG, Generali D, Ciruelos E, et al. European inter-institutional impact study of MammaPrint. Breast 2014;23:423-428.

20. Knauer M, Mook S, Rutgers EJ, et al. The predictive value of the 70-gene signature for adjuvant chemotherapy in early breast cancer. Breast Cancer Res Treat 2010;120:655-661.

21. Chen $Y$, Briesacher BA. Use of instrumental variable in prescription drug research with observational data: a systematic review. J Clin Epidemio/ 2011;64: 687-700.

22. Bueno-de-Mesquita JM, Linn SC, Keijzer R, et al. Validation of 70-gene prognosis signature in node-negative breast cancer. Breast Canc Res Treat 2009;117:483-95.

23. Buyse M, Loi S, van't Veer L, et al.; TRANSBIG Consortium. Validation and clinical utility of a 70-gene prognostic signature for women with node-negative breast cancer. J Nat/ Cancer Inst 2006;98:1183-1192

24. Wittner BS, Sgroi DC, Ryan PD, et al. Analysis of the MammaPrint breast cancer assay in a predominantly postmenopausal cohort. Clin Cancer Res 2008;14:2988-2993

25. Mook S, Schmidt MK, Weigelt B, et al. The 70-gene prognosis signature predicts early metastasis in breast cancer patients between 55 and 70 years of age. Ann Oncol 2010;21:717-722.

26. Straver ME, Glas AM, Hannemann J, et al. The 70-gene signature as a response predictor for neoadjuvant chemotherapy in breast cancer. Breast Cancer Res Treat 2010;119:551-558.

27. Esserman LJ, Berry DA, Cheang MC, et al.; I-SPY 1 TRIAL Investigators. Chemotherapy response and recurrence-free survival in neoadjuvant breast cancer depends on biomarker profiles: results from the I-SPY 1 TRIAL (CALGB 150007/150012; ACRIN 6657). Breast Cancer Res Treat 2012;132:1049-1062.

28. Whitworth P, Stork-Sloots L, de Snoo FA, et al. Chemosensitivity predicted by BluePrint 80-gene functional subtype and MammaPrint in the Prospective Neoadjuvant Breast Registry Symphony Trial (NBRST). Ann Surg Oncol 2014;21:3261-3267 